\title{
Accretion and Outflow Activity in the Earliest Phases of Star-Formation: CO, Sub-mm Continuum, and Near-Infrared Observations
}

\author{
K.-W. Hodapp \\ Institute for Astronomy, University of Hawaii, 2680 Woodlawn Drive, \\ Honolulu, HI 96822 \\ E. F. Ladd \\ Five College Radio Astronomy Observatory, University of \\ Massachusetts, 619 Lederle Graduate Research Center, Amherst, MA \\ 01003
}

\section{Introduction}

Stars in the earliest phases of their formation, i.e., those accreting the main component of their final mass, are deeply embedded within dense cores of dust and molecular material. Because of the high line-of-sight extinction and the large amount of circumstellar material, stellar emission is reprocessed by dust into long wavelength radiation, typically in the far-infrared and sub-millimeter bands. Consequently, the youngest sources are strong submillimeter continuum sources, and often undetectable as point sources in the near-infrared and optical. The most deeply embedded of these sources have been labelled "Class 0" sources by André, Ward-Thompson, \& Barsony (1994), in an extension of the spectral energy distribution classification scheme first proposed by Adams, Lada, \& Shu (1987).

These youngest sources are characterized by a low ratio of bolometric luminosity to submillimeter luminosity $\left(\mathrm{L}_{b o l} / \mathrm{L}_{1.3 m m} ;\right.$ André, Ward-Thompson, \& Barsony (1993), and a low bolometric temperature ( $T_{b o l}$; Myers \& Ladd 1993), both of which signify that the spectral energy distribution is narrow and peaks at long wavelengths. In addition, most if not all of these sources are associated with powerful and well-collimated outflows. The correlation between extreme youth and outflow activity is so well-established that the presence of an outflow is widely considered a requirement for Class 0 membership (Barsony 1994).

In this poster, we present narrow-band $\mathrm{H}_{2} \mathrm{~S}(1)+$ continuum images of several extremely young stars, with classifications ranging from Class 0 to Class I. We show that molecular hydrogen emission 1) is commonly associated with these young sources, 2) can be used to delineate the structure and orientation of their outflows, and 3) in some cases can confirm or call into question the association of outflows with embedded sources. Toward three sources presented in this contribution, we find evidence for new, previously undetected outflows. Toward all of the sources, we find evidence for multiple, probably independent, outflows suggesting that even at the earliest evolutionary stages, stars are typically associated in groups. 


\section{Conclusions}

We have studied the $\mathrm{H}_{2} \mathrm{~S}(1)$ line emission associated with the molecular outflows of extremely young stars. NGC 1333 IRAS 2, IRAS 4A and 4B, and IRAS $08076-3556$ are classified as class 0 sources. IRAS05173-0555 in L1634 and Cep E can best be described as transition objects between Class 0 and Class I (Hodapp \& Ladd, 1995; Ladd \& Hodapp, 1996).

Based on our current understanding of the evolutionary status corresponding to the spectral energy distribution classes, our sample contains objects in the process of accreting the bulk of their later stellar mass. We find at least one molecular outflow associated with each of the IRAS sources studied here, using morphological arguments. In many cases we find evidence for double outflows within the positional uncertainty of the IRAS sources. These multiple outflows must be coeval within the kinematic age of the outflows and the expected duration of the Class 0 phase - of order a few $10^{4}$ years (Barsony 1994).

We conclude that the known trend toward stars forming in groups or clusters on a timescale of $10^{6} \mathrm{yr}$ and with dozens or hundreds of members (Hodapp 1994) continues down to smaller scales of time, volume, and mass. Whether any of these double outflow sources are proto-binary stars cannot be decided.

We have discovered a substantial increase in the K-band brightness of a young, deeply embedded star in the Serpens NW star-forming region between 1994 August (Fig. 7) and 1995 July (Fig. 8). The photometric history suggests a similarity to FUor or EXor outbursts. The star is more deeply embedded than any previous examples of those two types of eruptive events, however. Even in its bright state the object is invisible in $\mathrm{J}$, only scattered radiation is seen in $\mathrm{H}$, the $\mathrm{K}$ band is still dominated by scattered light, and only in $\mathrm{L}^{\prime}$ and longer wavelengths do we see a pointlike source. The Serpens Deeply Embedded Outburst Star (DEOS) has been detected at $800 \mu \mathrm{m}$, confirming its very young age. Its spectrum (Fig. 9) between $2.0 \mu \mathrm{m}$ and $2.5 \mu \mathrm{m}$ is a very steep, pure continuum, steeper than any published spectrum of a young embedded star. Most likely, the spectrum is thermal radiation from dust enshrouding the young star that completely veils any of the spectral features characterizing less obscured FUors and EXors (Hodapp et al., 1996).

\section{References}

Adams, F. C., Lada, C. J., \& Shu, f. H. 1987, ApJ, 312, 788

André, Ph., Ward-Thompson, D., Barsony, M. 1993, ApJ, 406, 122

Barsony, M. 1994, Clouds, Cores, and Low Mass Stars, ASP Conference Series, Vol. 65, eds. D. P. Clemens and R. Barvainis, 197

Hodapp, K.-W. 1994, ApJS, 94, 615

Hodapp, K.-W., \& Ladd, E. F. 1995, ApJ 453, 715

Hodapp, K.-W., Hora, J. L., Rayner, J. T., Pickles, A. J., \& Ladd, E. F. 1996, ApJ 468,861

Ladd, E. F., \& Hodapp, K.-W. 1996, ApJ, in press

Myers, P. C., Ladd, E. F. 1993, ApJ, 413, L47 